\title{
Cysteine Cathepsins and Their Prognostic and Therapeutic Relevance in Leukemia
}

\author{
Mohit Arora ${ }^{1}$ Garima Pandey ${ }^{1}$ Shyam S. Chauhan ${ }^{1,2}$ \\ ${ }^{1}$ Department of Biochemistry, All India Institute of Medical \\ Sciences, New Delhi, India \\ 2Department of Biotechnology, All India Institute of Medical \\ Sciences, New Delhi, India

\begin{abstract}
Address for correspondence Shyam S. Chauhan, Department of Biotechnology, All India Institute of Medical Sciences, New Delhi 110029, India (e-mail: s_s_chauhan@hotmail.com).
\end{abstract}

\begin{abstract}
Cysteine cathepsins are lysosomal proteases that require Cys-His ion pair in their catalytic site for enzymatic activity. While their aberrant expression and oncogenic functions have been widely reported in solid tumors, recent findings suggest that these proteases also play an important role in the pathogenesis of hematological malignancies. In this review, we summarize the potential clinical implications of cysteine cathepsins as diagnostic and prognostic markers in leukemia, and present evidences which supports the utility of these proteases as potential therapeutic targets in hematological malignancies. We also highlight the available information on the expression patterns, regulation, and potential functions of cysteine cathepsins in normal hematopoiesis and hematological malignancies. In hematopoiesis, cysteine cathepsins play a variety of physiological roles including regulation of hematopoietic stem cell adhesion in the bone marrow, trafficking, and maturation. They are also involved in several functions of immune cells which include the selection of lymphocytes in the thymus, antigen processing, and presenta-

Keywords

- cathepsins

- leukemia

- proteases

- acute myeloid leukemia

- CTSL

- CTSB tion. However, the expression of cysteine cathepsins is dysregulated in hematological malignancies where they have been shown to play diverse functions. Interestingly, several pieces of evidence over the past few years have demonstrated overexpression of cathepsins in leukemia and their association with worst survival outcomes in patients. Strategies aimed at altering the expression, activity, and subcellular localization of these cathepsins are emerging as potential therapeutic modalaties in the management of hematological malignancies. Recent findings also suggest the involvement of these proteases in modulating the immune response in leukemia and lymphomas.
\end{abstract}

\section{Introduction}

Cysteine cathepsins are endo-lysosomal proteases that are primarily responsible for the turnover and degradation of intracellular proteins. These proteases are also involved in various other physiological processes including antigen presentation, ${ }^{1}$ sperm maturation, ${ }^{2}$ pro-hormone activation, ${ }^{3}$ ovulation, ${ }^{4}$ and fetal implantation. ${ }^{5,6}$ Altered expression of these proteases underlie various pathological processes such as atherosclerosis, ${ }^{7}$ rheumatoid arthritis, ${ }^{8}$ autoimmune disorders, ${ }^{9}$ and several types of malignancies. ${ }^{10,11}$

Cysteine cathepsins contain a highly conserved cysteine residue in their active sites. Due to a high degree of homology to the plant enzyme papain, these proteases have been

(c) 2021. National Academy of Medical Sciences (India).

This is an open access article published by Thieme under the terms of the Creative Commons Attribution-NonDerivative-NonCommercial-License, permitting copying and reproduction so long as the original work is given appropriate credit. Contents may not be used for commercial purposes, or adapted, remixed, transformed or built upon. (https://creativecommons.org/licenses/by-nc-nd/4.0/).

Thieme Medical and Scientific Publishers Pvt. Ltd. A-12, 2nd Floor, Sector 2, Noida-201301 UP, India 
included in the $\mathrm{C} 1$ family of the clan CA proteases. ${ }^{12}$ HUGO Gene Nomenclature Committee have recognized a total of 15 functional genes for cathepsins in the human genome (https://www.genenames.org/). These include 11 cysteine cathepsins (CTS-B, C, F, H, K, L, O, S, V, W, and Z) and four noncysteine cathepsins (CTS-A, G, D, and E). They are synthesized as catalytically inactive pre-pro-enzymes (zymogens) on endoplasmic reticulum (ER) bound ribosomes and acquire mannose 6-phosphate tag while passing through the ER-Golgi network. ${ }^{13}$ This tag along with the vesicular mannose 6-phosphate plays a key role in targeting them to lysosomes. In the acidic environment of lysosomes, these zymogens are proteolytically processed into their catalytically active form. The processed form of cysteine cathepsins ranging from 20 to $35 \mathrm{kDa}$ in size consists of a heavy and a light chain subunit which are linked by disulphide bridges. However, unlike other cysteine cathepsins, the processed form of CTSC exists as an oligomer. While most cathepsins are active at acidic pH, CTSS and CTSL display their optimal activities at alkaline and neutral $\mathrm{pH}$, respectively. ${ }^{14}$

Although a majority of human cysteine cathepsins (CTS-B, $\mathrm{H}, \mathrm{L}, \mathrm{C}, \mathrm{X}, \mathrm{F}, \mathrm{O}$, and V) are ubiquitously expressed, cathepsin K, $\mathrm{W}$, and $\mathrm{S}$ display tissue-specific pattern. ${ }^{14}$ CTSK is expressed in osteoclasts, most epithelial cells, and also in the synovial fibroblasts of rheumatoid arthritis patients, whereas CTSV (named as L2, a variant of CTSL) is found in thymus and testis. CTS such as K, L, S, F, V, and B which display high elastolytic and collagenolytic activities are involved in ECM remodeling. Since cathepsin B, C, H, and L are regulated by common transcription factors, they are often coexpressed in a variety of tissues and cell types. Studies using oligopeptide libraries have revealed overlapping substrate specificities of cysteine cathepsins but individual preferences to different peptide sequence. ${ }^{15,16}$

Elevated expression of cysteine cathepsins in solid tumors has been extensively documented. ${ }^{11,17}$ They promote tumor cell proliferation, inhibit apoptosis, and mediate metastasis. Higher enzymatic activities of cysteine cathepsins are also associated with poor overall survival outcomes in leukemic patients. ${ }^{18,19}$ In this review, we attempt to present current knowledge about the role of cysteine cathepsins in the pathogenesis of leukemia and their utility in its management.

\section{Cysteine Cathepsins in Hematopoiesis and Immune Cells Functions}

Under physiological conditions, a majority of cysteine cathepsins are primarily localized to lysosomes and play a key role in the turnover and degradation of intracellular proteins. However, localization of these proteases in the nucleus, cell surface, and extracellular milieu is also documented where they perform more specialized functions. ${ }^{14}$ Several cysteine cathepsins are expressed in cells at different stages of hematopoiesis. In the bone marrow, hematopoietic stem cells (HSCs) and other progenitor cells are trapped in complex microenvironment containing osteoblasts and stromal cells. This adhesion is partially mediated by chemoattractant CXCL12. ${ }^{20}$ Osteoblasts and stromal cells synthesize and secrete large quantities of CTSZ, which in turn degrade CXCL12 and thereby regulate hematopoietic stem and progenitor cell trafficking. ${ }^{21}$ Similarly, cathepsin S and D coordinate to facilitate the commitment of HSCs to differentiate into dendritic cells. ${ }^{22}$ Moreover, CTSZ has been shown to control phagocytosis in macrophages and maturation of dendritic cells via proteolytically activating $\beta 2$ integrin receptor Mac-1 (CD11b/CD18). ${ }^{23}$ Interestingly, Lalanne et al through a series of elegant experiments, established that toll-like receptor-9 (TLR-9) signaling inhibits expansion of pro-B cells via CTSB dependent apoptosis, thereby demonstrating the involvement of this cysteine cathepsin in the regulation of lymphopoiesis. ${ }^{24}$

Several studies demonstrating the role of cysteine cathepsins in antigen processing in both professional and nonprofessional antigen presenting cells have been reviewed previously. ${ }^{25,26}$ Multiple cathepsins are involved in invariant chain processing and major histocompatibility complex class II peptide loading by macrophages. ${ }^{27}$ During thymic selection, several proteases are differentially expressed in thymic antigen presenting cells to process autoantigens, which governs the selection of autoreactive T-cells. Cathepsin S, L, B, and D are involved in antigen processing in APCs. ${ }^{28} \mathrm{CTSV}$ is the dominant cysteine protease in cortical thymic epithelial cells, while CTSL and CTSS are restricted to dendritic and macrophage-like cells. ${ }^{29}$ Interestingly, CTSS plays a central role in the processing of the autoantigens in the thymus. ${ }^{30}$ However, CTSW, the predominant cathepsin of natural killer cells and $\mathrm{CD} 8^{+}$T-lymphocytes is secreted during cytotoxic $T$ cell effector functions. ${ }^{31}$ Recently, functions of cysteine cathepsins in tumor-associated immune cells have been reviewed in detail. ${ }^{32}$

\section{Prognostic Significance of Cysteine Cathepsins in Leukemia}

The elevated expression of cysteine cathepsins in pathological conditions such as inflammatory diseases, cancer cell proliferation, and metastasis has prompted researchers over the years to explore the clinical significance of these proteases. The utility of circulating cysteine cathepsins as a potential diagnostic biomarker has been explored in solid tumors such as nasopharyngeal, ${ }^{33}$ gastric, ${ }^{34}$ and lung cancer. ${ }^{35}$

In 2003, overexpression of cysteine cathepsin $\mathrm{W}$ and $\mathrm{C}$ was demonstrated in peripheral blood mononuclear cells (PBMCs) of large granular lymphocytic (LGL) leukemia patients. ${ }^{36}$ However, protease inhibitors such as cystatin C, A, alpha-1 antitrypsin, and metalloproteinase inhibitors were observed to be downregulated in these patients. A pilot study in our laboratory was undertaken to assess the expression of cysteine cathepsin B and L in the (PBMCs) of pediatric acute myeloid leukemia (AML) patients and age-matched healthy controls by real-time PCR, and enzymatic assays for both these proteases. ${ }^{37}$ The results of this study revealed significantly higher activity and expression of both these proteases in AML patients compared to the healthy controls. Furthermore, the expression of cystatin C displayed a strong negative correlation with the activities of both CTSB and 
CTSL in AML patients. Interestingly, AML patients with higher activities and expression of these cathepsins exhibited an inferior overall, and event-free survival as compared to the patients with lower activities of the same. These results suggested the prognostic significance of both CTSL and CTSB in pediatric AML. Subsequent studies were undertaken to validate these findings by using a large cohort of 101 newly diagnosed pediatric AML patients and 35 healthy controls. In these studies, the expression and activities of both the proteases were assessed by real-time PCR, Western blotting, and enzyme assays, respectively before the induction of chemotherapy and postinduction. A detailed plan of the same is summarized in $\boldsymbol{- F i g}$. 1. The results of these studies established significantly higher expression and activities of both these proteases not only in PBMCs but also in bone marrow mononuclear cells (BMMCs) of pediatric AML patients as compared to healthy controls. ${ }^{18,19}$ Activity of both the proteases displayed a dramatic reduction in response to chemotherapy, thereby suggesting the association of these proteases with the status of malignancy. By multivariate analysis, CTSL and CTSB in BMMCs emerged as independent prognostic markers for predicting the poor outcome of the disease. Survival analysis based on median CTSL/CTSB activity in pediatric AML patients is summarized in - Table $\mathbf{1} .^{18,19}$ As evident from this table, the overall survival (OS) and event-free survival (EFS) in AML patient group with low CTSL activity (lower than the median value) were observed to be 20.4 and 12.5 months, respectively. Whereas OS and EFS in patient group with high activity (higher than the median value) of this protease were observed to be 8.0 and 9.8 months, respectively. Similarly, OS and EFS in group of patients with low activity of CTSB (in PBMC) were estimated to be 26.2 and 14.3 months, respectively. On the other hand, OS and EFS in patients with high CTSB activity were found to be 8.23 months and 9.8 months, respectively. Thus these results conclusively demonstrated significantly higher OS and EFS in patients with low activities of CTSL and CTSB as compared to the patient group with high activity of these proteases. Similar findings were observed in this study when patients were divided based on the activity of these cysteine cathepsins in BMMCs. Thus, Pandey et al convincingly demonstrated significantly inferior OS and
EFS in AML patients with high CTSB and CTSL activities as compared to the low activity group, thereby establishing their utility as potential prognostic markers in this malignancy. ${ }^{18,19}$ Although the activities of these proteases were significantly higher in patient's BMMCs as compared to PBMCs, they displayed a strong positive correlation in both the cell types. These findings confirmed that the expression/activity of CTSB and CTSL in PBMCs reflects their expression in BMMCs of AML patients, which enhances the feasibility of assaying these proteases in peripheral blood samples for predicting the outcome of the disease or to monitor the progress of the therapy.

Chromosomal localization studies mapped human cathepsin L gene to 9q 21-22.38 Altered expression of genes located on the long arm ( $\mathrm{q}$ arm) of chromosomes 9 was previously demonstrated in chronic myelogenous leukemia (CML) patients. ${ }^{39}$ Given these reports and observed overexpression of CTSL in AML patients, Samaiya et al measured the activity and expression of this protease in the PBMCs of $47 \mathrm{CML}$ patients. ${ }^{40}$ In total, 37 adults with systemic infection as well as 50 healthy volunteers served as controls in this study. This investigation revealed significantly higher expression and activity of CTSL in CML patients compared to patients with systemic infection and healthy volunteers. However, CML patients in the accelerated phase/blast crisis (AP/BC) displayed a drastic decrease in CTSL expression and activity as compared to the patients in the chronic phase of the disease. Promoter methylation studies revealed that this drastic decrease in CTSL expression in $\mathrm{AP} / \mathrm{BC}$ patients was due to hypermethylation of its promoter. These findings were further corroborated by the treatment of human blast crisis cells K562 by DNA methyltransferase inhibitor 5-Azacytidine, which restored the expression of CTSL in these cells to the level of chronic phase. These results were further confirmed by the treatment of PBMCs isolated from blast crisis patients with 5-Azacytidine. In chronic phase patients, a CTSL expression exhibited a very strong positive correlation with the expression of vascular endothelial growth factor (VEGF). Thus, in view of the previous reports on transcriptional upregulation of CTSL expression by VEGF, ${ }^{41}$ elevated levels of CTSL in chronic phase patients were attributed to

Table 1 Survival analysis of pediatric acute myeloid leukemia patients by the median activity of cysteine cathepsins.

\begin{tabular}{|c|c|c|c|c|c|c|c|c|}
\hline & $\begin{array}{l}\text { CTSL } \\
\text { activity } \\
\text { PBMCs } \\
\text { (Low) }\end{array}$ & $\begin{array}{l}\text { CTSL } \\
\text { activity } \\
\text { PBMCs } \\
\text { (High) }\end{array}$ & $\begin{array}{l}\text { CTSL } \\
\text { activity } \\
\text { BMMCs } \\
\text { (Low) }\end{array}$ & $\begin{array}{l}\text { CTSL } \\
\text { activity } \\
\text { BMMCs } \\
\text { (High) }\end{array}$ & $\begin{array}{l}\text { CTSB } \\
\text { activity } \\
\text { PBMCs } \\
\text { (Low) }\end{array}$ & $\begin{array}{l}\text { CTSB } \\
\text { activity } \\
\text { PBMCs } \\
\text { (High) }\end{array}$ & $\begin{array}{l}\text { CTSB } \\
\text { activity } \\
\text { BMMCs } \\
\text { (Low) }\end{array}$ & $\begin{array}{l}\text { CTSB } \\
\text { activity } \\
\text { BMMCs } \\
\text { (High) }\end{array}$ \\
\hline $\mathrm{EFS}(\mathrm{mo})$ & $12.5^{\mathrm{a}}$ & 8.0 & $12.4^{a}$ & 7.9 & $14.3^{\mathrm{a}}$ & 8.23 & $13.8^{a}$ & 8.2 \\
\hline OS (mo) & $20.4^{a}$ & 9.8 & $18.1^{\mathrm{a}}$ & 9.4 & $26.2^{\mathrm{a}}$ & 9.8 & $26.2^{\mathrm{a}}$ & 9.5 \\
\hline References & \multicolumn{4}{|c|}{18} & \multicolumn{4}{|c|}{9} \\
\hline
\end{tabular}

Abbreviations: BMMC, bone marrow mononuclear cell; CTSB, cathepsin B; CTSL, cathepsin L; EFS, event free survival; OS, overall survival; PBMC, peripheral blood mononuclear cell.

Note: Peripheral blood mononuclear cells and bone marrow mononuclear cells were isolated by percol density gradient centrifugation from 103 newly diagnosed acute myeloid leukemia patients. The cells were lysed and the activities of CTSB and CTSL were assayed in the cell lysates. The median value of each of the above mentioned cysteine cathepsin was determined. Patients were divided into two groups based on the median value of enzyme activities. The patients with CTSB activities higher or equal to the median values were placed in the high enzyme activity group and rest in the low enzyme activity group. A similar criterion was used for placing patients in high or low CTSL groups. Kaplan-Meier survival analysis was used to determine the association of protease expression with event-free survival and overall survival. The relapse or death of the patient was considered as an event.

aValues significantly different from high cysteine cathepsin group. 
(A)

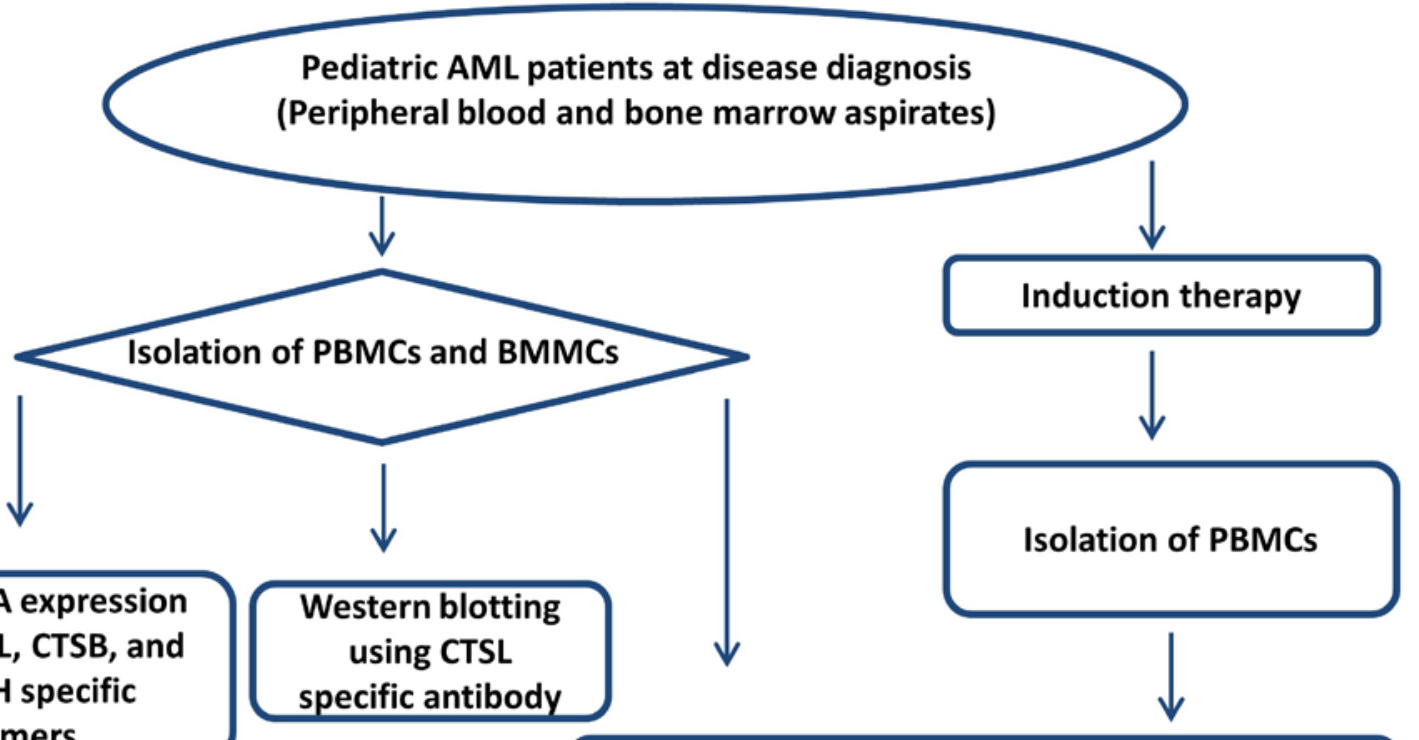

The mRNA expression using CTSL, CTSB, and GAPDH specific primers

Enzyme activity measurement using fluorogenic substrate CBZPhe-Arg-NMec

Statistical analysis and assessment of prognostic value (Kaplan-Meier survival analysis and Cox regression analysis)

(B)

Inhibition of CTSB activity in AML cell line, THP-1, using CA-074Me, a specific CTSB inhibitor<smiles>CCC</smiles>

\section{Effect on cell} survival : MTT assay

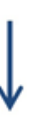

Effect on cell growth: Cell cycle analysis<smiles>[C]1C=C1</smiles>

Mode of cell death: Annexin V/PI staining and LDH assay

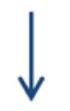

Involvement of caspases: Caspase- 8 and Caspase-3/7 enzyme assay

(C)

Effect/Interaction of cytosine arabinoside and doxorubicin on/with CTSB
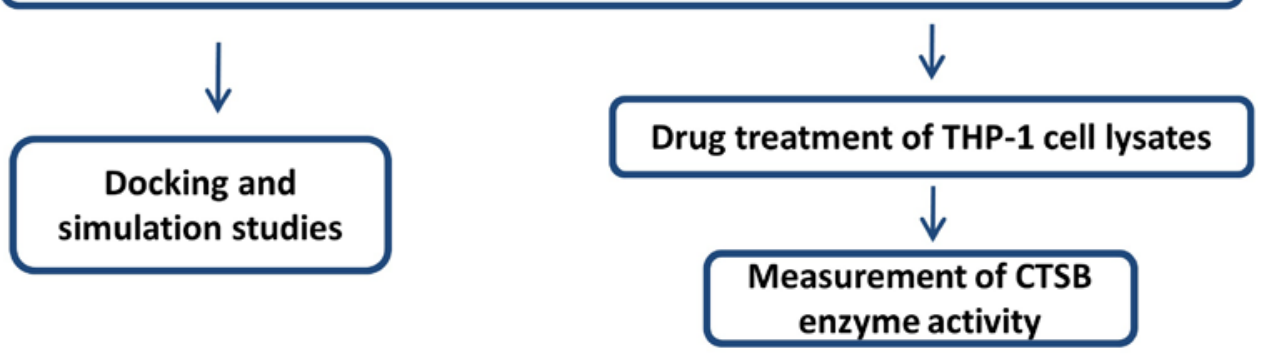

Fig. 1 (A-C) Experimental plan used to investigate the prognostic and therapeutic significance of cathepsins B and cathepsin L in acute myeloid leukemia. Figure edited and reproduced from Pandey et al. ${ }^{18,19}$ 
elevated expression of VEGF during this phase of malignancy. Elevation in CTSL expression by the treatment of PBMCs isolated from healthy volunteers with recombinant VEGF corroborated these findings. This study although did not evaluate the prognostic significance of CTSL, demonstrated that unlike in AML, this protease is overexpressed in CML patients in chronic phase and thus associated with better outcome of the disease. ${ }^{40}$

While the above mentioned studies strongly support prognostic implications of cysteine cathepsins in individual hematological malignancies, correlating the expression and activities of these proteases with clinical/molecular subtype may further confirm the utility of cysteine cathepsins as prognostic markers. In line with this view, Wrona et al compared the expression of CTSB in different genetic subtypes of B-cell precursor acute lymphoblastic leukemia (BCP-ALL) and observed overexpression of CTSB in BMMCs of BCP-ALL patients with deletion of paired box 5 (PAX5) gene, but no other primary genetic abnormality. ${ }^{42}$ However, this differential expression of CTSB did not have any significant impact on the 5-year recurrence-free survival in these patients.

In the past few years, several studies have explored the utility of cysteine cathepsins in diagnostic imaging for the detection of cancers. ${ }^{43}$ Activity-based fluorescence probes for these proteases have been successfully used for image-guided surgery of multiple cancers ${ }^{44,45}$ and bone diseases. ${ }^{46-48} \mathrm{~A}$ similar strategy might prove beneficial for less invasive in vivo detection of leukemic cells in the bone marrow.

Although fewer studies assessing the role of cysteine cathepsins in blood cancer are available as compared to solid tumors, they certainly provide a rationale to further explore their potential as diagnostic/prognostic markers in leukemia. Moreover, the systemic nature of leukemic cells may allow their usage as routine noninvasive biomarkers in clinical practice. Various studies which which have been carried out to investigate the utility of cysteine cathepsins as biomarkers in leukemia are listed in - Table 2 .

\section{Cathepsins as Therapeutic Targets in Leukemia}

Initial studies to understand the role of cysteine cathepsins in different pathologies by inhibition of their activities proved difficult because of the broad specificity of inhibitors. However, computational approaches eventually lead to the identification of specific inhibitors. The details of these inhibitors have been extensively reviewed. ${ }^{49}$ Active site mapping strategies combined with inhibitor library screenings lead to the identification of several specific inhibitors for CTSB and CTSL. ${ }^{50-52}$ Using in vitro and mouse xenograft models, several studies have demonstrated the potential therapeutic utility of cysteine cathepsin inhibition in the management of cancers of the pancreas, prostate, and brain. ${ }^{53-55} \mathrm{~A}$ combination of cysteine cathepsins with preexisting targeted therapies have also been reported to improve therapeutic effectiveness. For example, the combined use of anti-VEGF antibody and anti-cathepsin S antibody synergistically inhibits angiogenesis in solid tumors. ${ }^{56}$ Similarly, CTSS inhibitor (Z-FL-COCHO) sensitized renal carcinoma cells to tumor necrosis factor-related apoptosis-inducing ligand-mediated apoptosis in vitro. ${ }^{57}$ Interestingly, cysteine cathepsins are also expressed at high levels in stromal cells of the tumor microenvironment (TME), particularly in tumor-associated macrophages (TAMs). TAMs exhibit high plasticity in their function and display both pro- and antitumor properties depending upon cues received from the

Table 2 Studies on potential utility of cysteine cathepsins as biomarkers in leukemia

\begin{tabular}{|c|c|c|c|c|}
\hline Sample/Tissue & Detection & Method & $\begin{array}{l}\text { Feasibility of } \\
\text { implementation in clinical } \\
\text { practice }\end{array}$ & Reference(s) \\
\hline \multirow[t]{4}{*}{ BMMC/PBMC } & Total mRNA & qRT-PCR & Feasible and rapid & 19 \\
\hline & Cell specific mRNA level & $\begin{array}{l}\text { Cell enrichment by FACS/ } \\
\text { MACS followed by qRT-PCR }\end{array}$ & $\begin{array}{l}\text { Less feasible in the routine } \\
\text { clinical setting }\end{array}$ & NA \\
\hline & Cell specific protein level & Flow cytometry & $\begin{array}{l}\text { Already used in the routine } \\
\text { clinical setting }\end{array}$ & 70 \\
\hline & Enzyme activity & Biochemical assay & $\begin{array}{l}\text { Feasible for some cathepsins, } \\
\text { including CTSB and CTSL }\end{array}$ & 18 \\
\hline Bone marrow cells & Enzyme activity & $\begin{array}{l}\text { In vivo imaging using activi- } \\
\text { ty-based probes }\end{array}$ & Potentially feasible & 44,45 \\
\hline \multirow[t]{2}{*}{ Serum/body fluids } & $\begin{array}{l}\text { Circulating total cathepsin } \\
\text { level }\end{array}$ & ELISA & Feasible and rapid & 71 \\
\hline & $\begin{array}{l}\text { Cathepsin/cystatin complex } \\
\text { level }\end{array}$ & $\begin{array}{l}\text { Sandwich ELISA with simulta- } \\
\text { neous recognition of cathep- } \\
\text { sin and cystatin present as } \\
\text { complex }\end{array}$ & Feasible and rapid & 72 \\
\hline
\end{tabular}

Abbreviations: BMMC, bone marrow mononuclear cell; CTSB, cathepsin B; CTSL, cathepsin L; ELISA, enzyme-linked immunosorbent assay; FACS, fluorescence activated cell sorting; MACS, magnetic activated cell sorting; NA, not available; PBMC, peripheral blood mononuclear cell; qRT-PCR, quantitative real time polymerase chain reaction. 

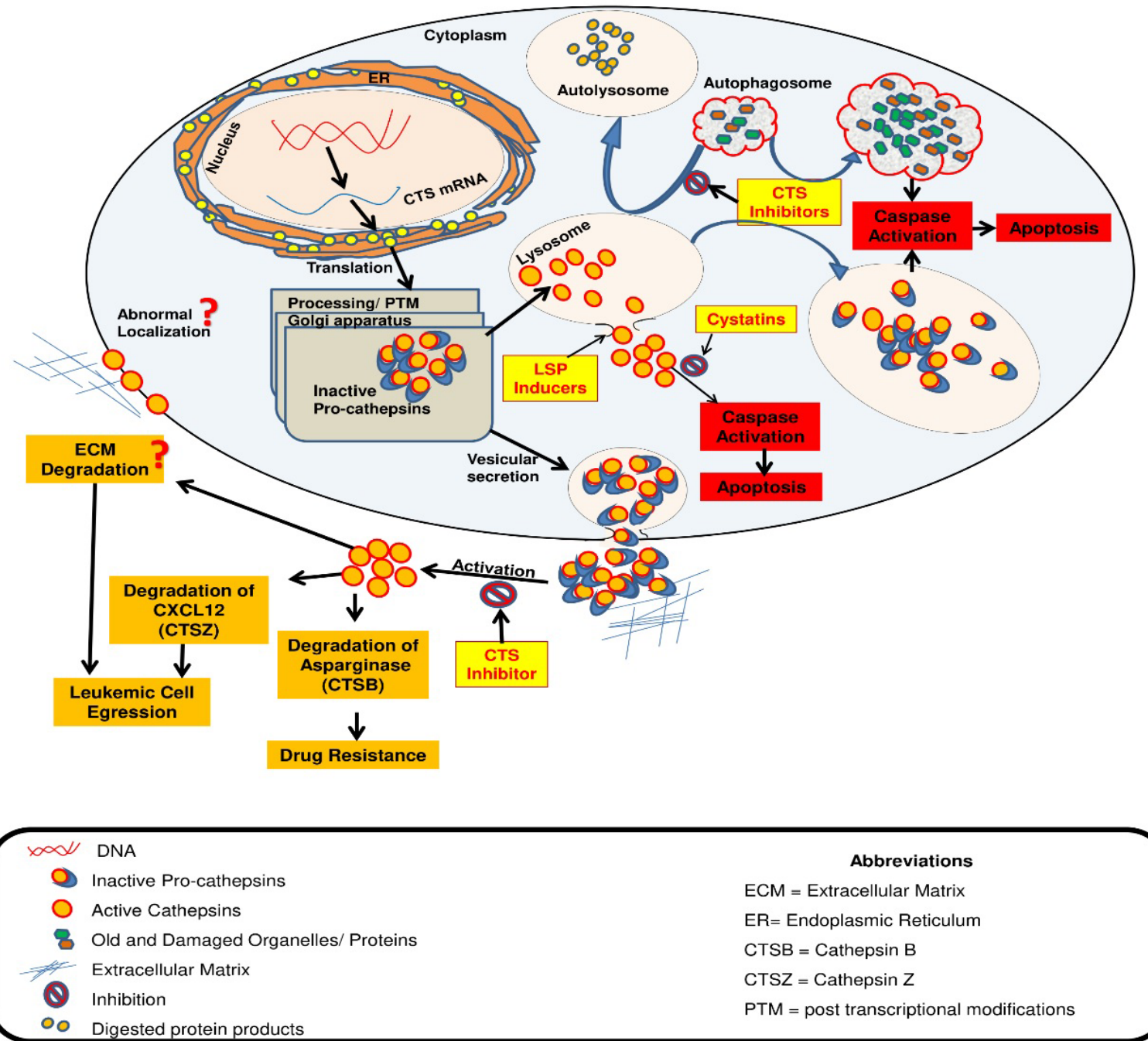

\author{
Abbreviations \\ $\mathrm{ECM}=$ Extracellular Matrix \\ $\mathrm{ER}=$ Endoplasmic Reticulum \\ $\mathrm{CTSB}=$ Cathepsin $\mathrm{B}$ \\ CTSZ $=$ Cathepsin $Z$ \\ PTM $=$ post transcriptional modifications
}

Fig. 2 Putative role of cysteine cathepsins in leukemia. The mRNA of cysteine cathepsins are synthesized in the nucleus, transported to the cytosol, and translated into pre-pro-cathepsins on ER bound ribosomes. These pre-pro-cathepsins further undergo post-translational modifications in the ER-Golgi network to form pro-cathepsins. These pro-cathepsins are either packaged into lysosomes or secreted out of the cells. In lysosomes or acidic leukemic microenvironment, these pro-cathepsins are proteolytically processed to their enzymatically active forms, where they function in the degradation and turnover of intracellular proteins and extracellular matrix, respectively. During protein degradation, autophagic phagosomes fuse with lysosomes (forming autophagosomes), where active cathepsin degrade these dead/damaged organelles and proteins for recycling. As cathepsins are overexpressed in leukemia, treatment with cathepsin inhibitors leads to the accumulation of cellular debris and enlarged lysosomes with inactive cathepsins, and subsequently, induce caspase-mediated apoptosis. Further, it may also inhibit the degradation of asparaginase and CXCL12 in the leukemic microenvironment, thereby regulating the egression and drug response of leukemic cells. Lysosome permeabilization inducers disrupt lysosomal membrane integrity leading to the release of cathepsins into the cytosol, which also induces apoptosis. Cystatins are endogenous inhibitors of cysteine cathepsins and protect cells from cytosolic cathepsin induced apoptosis. LSPs, lysosome permeabilization.

cellular microenvironment. ${ }^{58}$ Therefore, an important consideration for cysteine cathepsin inhibition may also be to first determine the cell-specific role of these proteases in the TME. In line with this, a study using a murine model of breast cancer reported that CTSB overexpression in tumor cells, not stromal cells to be associated with tumor progression. ${ }^{59}$ The secretion of cysteine cathepsins by TAMs has also been demonstrated to induce resistance to taxol, doxorubicin, and etoposide in breast cancer. ${ }^{60}$ Inhibition of cysteine cathepsins B, L, and S by a novel inhibitor GB111-NH2 has been shown to induce oxidative stress and apoptosis in macrophages ${ }^{61}$ Interestingly, apoptosis in TAMs also induced death of neighboring breast cancer cells in vivo and hence resulted in regression of the primary growth. In leukemia, the determination of the cellular origin of protease expression could be comparatively easier than solid tumors because of the ready availability of single cells suspensions. Additionally, the implementation of cysteine cathepsins as prognostic markers or in predicting the therapeutic response in future might be comparatively easier in leukemia than in solid tumors since flow cytometry is routinely used for the diagnosis of of this malignancy. It may further facilitate determination of their cell specific 
expression pattern along with established clinical markers and thus help in identification of molecular subtypes of leukemia.

Previous studies have demonstrated CTSB-mediated degradation of asparaginase, a drug used to treat childhood ALL, thereby rendering it ineffective in the treatment of this leukemia. ${ }^{62,63}$ This observation was further supported by increased half-life of asparaginase in patients harboring germline mutation in the CTSB gene, resulting in the deletion of a highly conserved lysine residue in its carboxy terminus (p.K237del).62 This may explain the extensive variations in response to asparaginase therapy observed in leukemic patients and suggests that CTSB inhibition may augment therapeutic response and overcome drug resistance in ALL.

Few studies have been undertaken to elucidate the mechanism of cysteine cathepsin inhibition mediated cell death in leukemia. Zhu and Uckun demonstrated induction of apoptosis in multiple human leukemia and lymphoma cell lines by the inhibition of cysteine cathepsins with pan cathepsin inhibitor I (CATI-I) ${ }^{64}$ This selective inhibitor of cysteine cathepsins triggered apoptosis in a caspase-independent, p53-independent, BAX-independent as well as MAP kinaseindependent fashion. With the recent advancements in the understanding of cell death pathways, determination of the full potential of targeting individual cysteine cathepsins may prove useful in the management of leukemia. With this objective, Pandey et al sought to investigate the therapeutic potential of CTSB inhibition in AML using an AML derived human cells line THP- $1 .{ }^{19}$ The strategy used in this study is given in - Fig. 1B, C. The results of this study conclusively demonstrated the abrogation of cell proliferation and induction of caspase-dependent apoptosis in THP-1 cells by inhibition of CTSB with CA-074 Me. ${ }^{19}$ Furthermore, doxorubicin-a conventional drug used to treat this malignancy-efficiently inhibited CTSB activity in vivo as well as in vitro, thereby suggesting that this therapeutic agent imparts some part of its cytotoxic effects on leukemic cells by inhibiting CTSB. Docking and simulation studies confirmed the binding of doxorubicin to the active site of CTSB with higher binding energy than its specific inhibitor CA-074 Me. These results establish the potential utility of CTSB as a therapeutic target. Consistent with these findings, benzyl-oxycarbonyl-phenylalanine-alanine-chloromethyl ketone (z-FA-CMK), a specific CTSB inhibitor has recently been shown to induce oxidative stress mediated apoptosis in leukemic T-cells at low concentrations, while at high concentrations, it induced necrotic cell death. ${ }^{65,66}$ The putative roles of cysteine cathepsins in leukemia have been summarized in

-Fig. 2.

In addition of being therapeutic targets in leukemia, cysteine cathepsins have also been shown to be mediators of cell death in certain conditions. Ivanov et al observed that certain CD20-specific mAbs and HLA-DR specific $\mathrm{mAb}$ induce a nonapoptotic cell death that is rapid, nonautophagic, and involve disintegration of lysosomal membrane, thereby releasing its contents including CTSB into the cytoplasm. ${ }^{67}$ Similarly, in another study, Puissant et al demonstrated that imatinib induced death of CML cells is associated with lysosomal membrane permeabilization leading to release of CTSB into the cytosolic compartment which in turn cleaves BCR-ABL and thereby induces apoptosis. ${ }^{68}$ Consistent with these findings, treatment of chronic lymphocytic leukemia cells with lysosomotropic agents also induced cell death following CTSB release. ${ }^{69}$

In summary, elevated levels of cysteine cathepsins in different types of leukemia and their association with poor survival outcomes in patients suggest their potential utility as a prognostic marker. Additionally, targeting specific cysteine cathepsins in combination with existing targeted therapies in leukemia may improve patient survival. Therefore, future studies aimed at delineating the cathepsin-mediated cell survival/apoptotic pathways in leukemia may open new therapeutic avenues.

\section{Funding}

Research in the laboratory of SSC is supported by research grants from Department of Science and Technology (India), Defence Research and Development Organisation (India), and Indian Council of Medical Research (India). M.A. is the recipient of SRF from Council of Scientific and Industrial Research (India).

\section{Conflict of Interest}

None declared.

\section{References}

1 Conus S, Simon HU. Cathepsins and their involvement in immune responses. Swiss Med Wkly 2010;140(2930):w13042

2 Mathur PP, Grima J, Mo MY, et al. Differential expression of multiple cathepsin mRNAs in the rat testis during maturation and following lonidamine induced tissue restructuring. Biochem Mol Biol Int 1997;42(2):217-233

3 Hook V, Funkelstein L, Lu D, Bark S, Wegrzyn J, Hwang S-R. Proteases for processing proneuropeptides into peptide neurotransmitters and hormones. Annu Rev Pharmacol Toxicol 2008;48:393-423

4 Carnevali O, Cionna C, Tosti L, Lubzens E, Maradonna F. Role of cathepsins in ovarian follicle growth and maturation. Gen Comp Endocrinol 2006;146(3):195-203

5 Amarante-Paffaro AM, Hoshida MS, Yokota S, et al. Localization of cathepsins D and B at the maternal-fetal interface and the invasiveness of the trophoblast during the postimplantation period in the mouse. Cells Tissues Organs 2011;193(6):417-425

6 Divya CP, Mahajan VS, Datta Gupta S, Chauhan SS. Differential activity of cathepsin L in human placenta at two different stages of gestation. Placenta 2002;23(1):59-64

7 Zhao CF, Herrington DM. The function of cathepsins B, D, and $\mathrm{X}$ in atherosclerosis. Am J Cardiovasc Dis 2016;6(4):163-170

8 Hashimoto Y, Kakegawa H, Narita Y, et al. Significance of cathep$\sin \mathrm{B}$ accumulation in synovial fluid of rheumatoid arthritis. Biochem Biophys Res Commun 2001;283(2):334-339

9 Edman MC, Janga SR, Meng Z, et al. Increased Cathepsin S activity associated with decreased protease inhibitory capacity contributes to altered tear proteins in Sjögren's Syndrome patients. Sci Rep 2018;8(1):11044

10 Unraveling the Role of Proteases in Cancer Koblinski J, Ahram M, Sloane B. 2000;291 
11 Chauhan SS, Goldstein LJ, Gottesman MM. Expression of cathep$\sin$ L in human tumors. Cancer Res 1991;51(5):1478-1481

12 Rawlings ND, Barrett AJ, Bateman A. MEROPS: the database of proteolytic enzymes, their substrates and inhibitors. Nucleic Acids Res 2012;40(Database issue):D343-D350

13 Braulke T, Bonifacino JS. Sorting of lysosomal proteins. Biochim Biophys Acta 2009;1793(4):605-614

14 Turk V, Stoka V, Vasiljeva O, et al. Cysteine cathepsins: from structure, function and regulation to new frontiers. Biochim Biophys Acta 2012;1824(1):68-88

15 Choe Y, Leonetti F, Greenbaum DC, et al. Substrate profiling of cysteine proteases using a combinatorial peptide library identifies functionally unique specificities. J Biol Chem 2006;281(18):12824-12832

16 Biniossek ML, Nägler DK, Becker-Pauly C, Schilling O. Proteomic identification of protease cleavage sites characterizes prime and non-prime specificity of cysteine cathepsins B, L, and S. J Proteome Res 2011;10(12):5363-5373

17 Rudzińska M, Parodi A, Soond SM, et al. The role of cysteine cathepsins in cancer progression and drug resistance. Int J Mol Sci 2019;20(14):3602

18 Pandey G, Bakhshi S, Thakur B, Jain P, Chauhan SS. Prognostic significance of cathepsin L expression in pediatric acute myeloid leukemia. Leuk Lymphoma 2018;59(9):2175-2187

19 Pandey G, Bakhshi S, Kumar M, et al. Prognostic and therapeutic relevance of cathepsin B in pediatric acute myeloid leukemia. Am J Cancer Res 2019;9(12):2634-2649

20 Sugiyama T, Kohara H, Noda M, Nagasawa T. Maintenance of the hematopoietic stem cell pool by CXCL12-CXCR4 chemokine signaling in bone marrow stromal cell niches. Immunity 2006;25(6):977-988

21 Staudt ND, Aicher WK, Kalbacher H, et al. Cathepsin X is secreted by human osteoblasts, digests CXCL-12 and impairs adhesion of hematopoietic stem and progenitor cells to osteoblasts. Haematologica 2010;95(9):1452-1460

22 Martino S, Tiribuzi R, Ciraci E, et al. Coordinated involvement of cathepsins S, D and cystatin C in the commitment of hematopoietic stem cells to dendritic cells. Int J Biochem Cell Biol 2011;43(5):775-783

23 Kos J, Jevnikar Z, Obermajer N. The role of cathepsin X in cell signaling. Cell Adhes Migr 2009;3(2):164-166

24 Lalanne AI, Moraga I, Hao Y, et al. CpG inhibits pro-B cell expansion through a cathepsin B-dependent mechanism. J Immunol 2010;184(10):5678-5685

25 Honey K, Rudensky AY. Lysosomal cysteine proteases regulate antigen presentation. Nat Rev Immunol 2003;3(6):472-482

26 Hsing LC, Rudensky AY. The lysosomal cysteine proteases in MHC class II antigen presentation. Immunol Rev 2005;207(1):229-241

27 Shi G-P, Bryant RA, Riese R, et al. Role for cathepsin $F$ in invariant chain processing and major histocompatibility complex class II peptide loading by macrophages. J Exp Med 2000;191(7):1177-1186

28 Driessen C, Lennon-Duménil A-M, Ploegh HL. Individual cathepsins degrade immune complexes internalized by antigen-presenting cells via Fcgamma receptors. Eur J Immunol 2001;31(5):1592-1601

29 Tolosa E, Li W, Yasuda Y, et al. Cathepsin V is involved in the degradation of invariant chain in human thymus and is overexpressed in myasthenia gravis. J Clin Invest 2003;112(4):517-526

30 Stoeckle C, Quecke P, Rückrich T, et al. Cathepsin S dominates autoantigen processing in human thymic dendritic cells. J Autoimmun 2012;38(4):332-343
31 Stoeckle C, Gouttefangeas C, Hammer M, Weber E, Melms A, Tolosa E. Cathepsin $\mathrm{W}$ expressed exclusively in CD8+ T cells and NK cells, is secreted during target cell killing but is not essential for cytotoxicity in human CTLs. Exp Hematol 2009;37(2):266-275

32 Jakoš T, Pišlar A, Jewett A, Kos J. Cysteine cathepsins in tumor-associated immune cells. Front Immunol 2019;10:2037

33 Tan G, Liu Q, Tang X, et al. Diagnostic values of serum cathep$\sin \mathrm{B}$ and $\mathrm{D}$ in patients with nasopharyngeal carcinoma. BMC Cancer 2016;16:241

34 Liu W-L, Liu D, Cheng K, et al. Evaluating the diagnostic and prognostic value of circulating cathepsin $\mathrm{S}$ in gastric cancer. Oncotarget 2016;7(19):28124-28138

35 Kehlet SN, Bager CL, Willumsen N, et al. Cathepsin-S degraded decorin are elevated in fibrotic lung disorders - development and biological validation of a new serum biomarker. BMC Pulm Med 2017;17(1):110

36 Kothapalli R, Bailey RD, Kusmartseva I. Mane S, EplingBurnette PK, Loughran TP Jr. Constitutive expression of cytotoxic proteases and down-regulation of protease inhibitors in LGL leukemia. Int J Oncol 2003;22(1):33-39

37 Jain M, Bakhshi S, Shukla AA, Chauhan SS. Cathepsins B and $L$ in peripheral blood mononuclear cells of pediatric acute myeloid leukemia: potential poor prognostic markers. Ann Hematol 2010;89(12):1223-1232

38 Chauhan SS, Popescu NC, Ray D, Fleischmann R, Gottesman MM, Troen BR. Cloning, genomic organization, and chromosomal localization of human cathepsin L. J Biol Chem 1993;268(2):1039-1045

39 Albano F, Specchia G, Anelli L, et al. Genomic deletions on other chromosomes involved in variant $\mathrm{t}(9 ; 22)$ chronic myeloid leukemia cases. Genes Chromosomes Cancer 2003;36(4):353-360

40 Samaiya M, Bakhshi S, Shukla AA, Kumar L, Chauhan SS. Epigenetic regulation of cathepsin $\mathrm{L}$ expression in chronic myeloid leukaemia. J Cell Mol Med 2011;15(10):2189-2199

41 Keerthivasan S, Keerthivasan G, Mittal S, Chauhan SS. Transcriptional upregulation of human cathepsin L by VEGF in glioblastoma cells. Gene 2007;399(2):129-136

42 Wrona E, Jakubowska J, Pawlik B, et al. Gene expression of ASNS, LGMN and CTSB is elevated in a subgroup of childhood BCP-ALL with PAX5 deletion. Oncol Lett 2019; 18(6):6926-6932

43 Löser R, Pietzsch J. Cysteine cathepsins: their role in tumor progression and recent trends in the development of imaging probes. Front Chem 2015;3:37

44 Habibollahi P, Figueiredo J-L, Heidari P, et al. Optical imaging with a cathepsin $\mathrm{B}$ activated probe for the enhanced detection of esophageal adenocarcinoma by dual channel fluorescent upper GI endoscopy. Theranostics 2012;2(2):227-234

45 Ma W, Ma L, Zhe H, et al. Detection of esophageal squamous cell carcinoma by cathepsin B activity in nude mice. PLoS ONE 2014;9(3):e92351

46 Kozloff KM, Quinti L, Patntirapong S, et al. Non-invasive optical detection of cathepsin K-mediated fluorescence reveals osteoclast activity in vitro and in vivo. Bone 2009;44(2):190-198

47 Lai W-FT, Chang C-H, Tang Y, Bronson R, Tung C-H. Early diagnosis of osteoarthritis using cathepsin B sensitive near-infrared fluorescent probes. Osteoarthritis Cartilage 2004;12(3):239-244

48 Scales HE, Ierna M, Smith KM, et al. Assessment of murine collagen-induced arthritis by longitudinal non-invasive duplexed molecular optical imaging. Rheumatology (Oxford) 2016;55(3):564-572 
49 Li Y-Y, Fang J, Ao G-Z. Cathepsin B and L inhibitors: a patent review (2010 - present). Expert Opin Ther Pat 2017; 27(6):643-656

50 Schmitz J, Gilberg E, Löser R, Bajorath J, Bartz U, Gütschow M. Cathepsin B: active site mapping with peptidic substrates and inhibitors. Bioorg Med Chem 2019;27(1):1-15

51 Poreba M, Rut W, Vizovisek M, et al. Selective imaging of cathepsin L in breast cancer by fluorescent activity-based probes. Chem Sci (Camb) 2018;9(8):2113-2129

52 Yuan L, Sheng L, He W, et al. Discovery of novel cathepsin inhibitors with potent anti-metastatic effects in breast cancer cells. Bioorg Chem 2018;81:672-680

53 Nalla AK, Gorantla B, Gondi CS, Lakka SS, Rao JS. Targeting MMP-9, uPAR, and cathepsin B inhibits invasion, migration and activates apoptosis in prostate cancer cells. Cancer Gene Ther 2010;17(9):599-613

54 Jung M, Lee J, Seo H-Y, Lim JS, Kim E-K. Cathepsin inhibition-induced lysosomal dysfunction enhances pancreatic beta-cell apoptosis in high glucose. PLoS One 2015;10(1):e0116972

55 Soond SM, Kozhevnikova MV, Townsend PA, Zamyatnin AA Jr. Cysteine cathepsin protease inhibition: an update on its diagnostic, prognostic and therapeutic potential in cancer. Pharmaceuticals (Basel) 2019;12(2):87

56 Ward C, Kuehn D, Burden RE, et al. Antibody targeting of cathepsin S inhibits angiogenesis and synergistically enhances anti-VEGF. PLoS One 2010;5(9):e12543

57 Seo BR, Min K-J, Woo SM, et al. Inhibition of cathepsin S induces mitochondrial ROS that sensitizes TRAIL-mediated apoptosis through p53-mediated downregulation of Bcl-2 and c-FLIP. Antioxid Redox Signal 2017;27(4):215-233

58 Chen Y, Song Y, Du W, Gong L, Chang H, Zou Z. Tumorassociated macrophages: an accomplice in solid tumor progression. J Biomed Sci 2019;26(1):78

59 Bengsch F, Buck A, Günther SC, et al. Cell type-dependent pathogenic functions of overexpressed human cathepsin $B$ in murine breast cancer progression. Oncogene 2014;33(36):4474-4484

60 Shree T, Olson OC, Elie BT, et al. Macrophages and cathepsin proteases blunt chemotherapeutic response in breast cancer. Genes Dev 2011;25(23):2465-2479

61 Salpeter SJ, Pozniak Y, Merquiol E, Ben-Nun Y, Geiger T, Blum G. A novel cysteine cathepsin inhibitor yields macrophage cell death and mammary tumor regression. Oncogene 2015;34(50):6066-6078

62 van der Meer LT, Waanders E, Levers M, et al. A germ line mutation in cathepsin B points toward a role in asparaginase pharmacokinetics. Blood 2014;124(19):3027-3029

63 Patel N, Krishnan S, Offman MN, et al. A dyad of lymphoblastic lysosomal cysteine proteases degrades the antileukemic drug L-asparaginase. J Clin Invest 2009;119(7):1964-1973

64 Zhu D-M, Uckun FM. Cathepsin inhibition induces apoptotic death in human leukemia and lymphoma cells. Leuk Lymphoma 2000;39(3-4):343-354

65 Liow KY, Chow SC. The cathepsin B inhibitor z-FA-CMK induces cell death in leukemic $T$ cells via oxidative stress. Naunyn Schmiedebergs Arch Pharmacol 2018;391(1):71-82

66 Liow KY, Chow SC. The cathepsin B inhibitor, z-FA-CMK is toxic and readily induced cell death in human T lymphocytes. Toxicol Appl Pharmacol 2013;272(3):559-567

67 Ivanov A, Beers SA, Walshe CA, et al. Monoclonal antibodies directed to CD20 and HLA-DR can elicit homotypic adhesion followed by lysosome-mediated cell death in human lymphoma and leukemia cells. J Clin Invest 2009;119(8):2143-2159

68 Puissant A, Dufies M, Raynaud S, Cassuto J-P, Auberger P. Targeting lysosomes to eradicate imatinib-resistant chronic myelogenous leukemia cells. Leukemia 2010;24(5):1099-1101

69 Yoon J-Y, Szwajcer D, Ishdorj G, et al. Synergistic apoptotic response between valproic acid and fludarabine in chronic lymphocytic leukaemia (CLL) cells involves the lysosomal protease cathepsin B. Blood Cancer J 2013;3(10):e153-e153

70 Zhang M, Sukhumalchandra P, Enyenihi AA, et al. A novel HLA-A*0201 restricted peptide derived from cathepsin $G$ is an effective immunotherapeutic target in acute myeloid leukemia. Clin Cancer Res 2013;19(1):247-257

71 Monsouvanh A, Proungvitaya T, Limpaiboon T, et al. Serum cathepsin $B$ to cystatin $C$ ratio as a potential marker for the diagnosis of cholangiocarcinoma. Asian Pac J Cancer Prev 2014;15(21):9511-9515

72 Zore I, Krasovec M, Cimerman N, et al. Cathepsin B/cystatin C complex levels in sera from patients with lung and colorectal cancer. Biol Chem 2001;382(5):805-810 